\title{
Associations for Citizen Science: Regional Knowledge, Global Collaboration
}

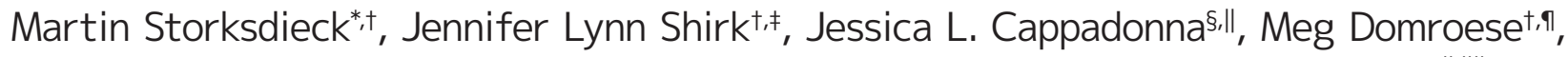

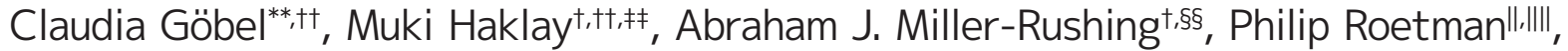 \\ Carla Sbrocchill,nq and Katrin Vohland*,,+十
}

Since 2012, three organizations advancing the work of citizen science practitioners have arisen in different regions: The primarily US-based but globally open Citizen Science Association (CSA), the European Citizen Science Association (ECSA), and the Australian Citizen Science Association (ACSA). These associations are moving rapidly to establish themselves and to develop inter-association collaborations. We consider the factors driving this emergence and the significance of this trend for citizen science as a field of practice, as an area of scholarship, and for the culture of scientific research itself.

Citizen science has attracted mainstream attention over the past three years. High-level events, funding, reports, and policies have emerged from many places around the world including the US White House, the Australian chief scientist, and the European Union. Even the term has been recognized as common vernacular. In 2014 the Oxford English Dictionary was the first dictionary to include citizen science, defining it as "scientific work undertaken by members of the general public, often in collaboration with or under the direction of professional scientists and scientific institutions." And three major membership organizations, organized as associations, have now formed to advance excellence in the development and management of citizen science and to support the growing and interconnected communities that have developed

\footnotetext{
* Center for Research on Lifelong STEM Learning, Oregon State University, Corvalis, OR, USA

Citizen Science Association (CSA), USA

₹ Cornell Lab of Ornithology, Ithaca, NY, US

§ Queensland University of Technology, Brisbane, QLD, Australia

" Australian Citizen Science Association (ACSA), Australia

" Gathering Waters: Wisconsin's Alliance for Land Trusts, Madison, WI, USA

${ }^{* *}$ Museum für Naturkunde Berlin (MfN), Berlin, Germany

${ }^{\dagger t}$ European Citizen Science Association (ECSA), \% Museum für Naturkunde Berlin, Germany

f^Department of Civil, Environmental and Geomatic Engineering, University College London, London, UK

§§ US National Park Service, Acadia National Park, Bar Harbor, ME, USA

III Barbara Hardy Institute, University of South Australia, Adelaide, SA, Australia

"Inniversity of Technology, Sydney, NSW, Australia

Corresponding author: Martin Storksdieck

(storksdieck@oregonstate.edu)
}

around the citizen science concept and practice. These are the Citizen Science Association (CSA), the European Citizen Science Association (ECSA), and the Australian Citizen Science Association (ACSA).

Establishment of these associations represents a milestone in the development of citizen science as a field, not just a research method or a form of organized engagement with science. Recognizing that the rapid growth and innovation in the field have, temporarily, outpaced opportunities to advance collaborative learning, the associations are engaging and supporting emerging practices for project design. While a burgeoning scholarship and professionalism around the practices and impacts of citizen science is flourishing along with the growth of the field, promising practices are widely dispersed across journals or even going unpublished; many practitioners have few opportunities to connect with and learn from colleagues doing similar work in other disciplines or locations; and skeptics of citizen science have insufficient awareness about the field to review proposals, tenure packets, or journal publications in a fair manner. The associations have formed to support a shared understanding of the opportunities, evidence-based practices, successes, challenges, and emerging standards of expectation for excellence in citizen science, and to help those within and beyond the field realize opportunities to appreciate or effectively build on the momentum gathering around citizen science as an accepted approach for research, outreach, and informal and formal education.

Membership associations are created in both professional fields and serious leisure/hobbyist endeavors at the crucial time of transition from emergence to established practice, the point when the need arises to codify practices, address shared concerns, and create systematic opportunities for networking among practitioners (Stebbins 1992). For example, associations for citizen science can become 
hubs for sharing the existing knowledge on citizen science practice and can provide support for mutual learning among practitioners, helping to inform effective use of citizen science initiatives and to identify and address common challenges across the field. In doing so, associations foster practice-focused connections across disciplines to help maximize impact, avoid duplication, inform strategic investment, and foster quality in all aspects of programming. The new citizen science associations are emerging as key institutions to spearhead outreach and education about citizen science for scientific peers, policy makers, and the public, establishing an identity and respected reputation for the field, and setting agendas for global discussions and discourses around the value and contribution of citizen science to solving global problems. Furthermore, these three associations (and those that are arising in other areas) will provide the institutional backbone and focus for citizen science as an academic endeavor, creating research agendas and providing avenues for research to influence practice irrespective of national boundaries, while acknowledging elements that are dependent on local culture, funding, and government structures.

\section{Significance of associations}

For citizen science as a field of practice: Even with great diversity across the landscape of citizen science projects (see Shirk et al. 2012 for one overview of varied traditions, approaches, and terminologies), project leaders face many common challenges, and project innovations that overcome these challenges can often transfer across boundaries. One particular challenge for practitioners of this highly interdisciplinary practice (individuals who design, manage, support, and/or conduct research through citizen science) is that work is often done in isolation. Consequently, too many citizen science projects still "reinvent the wheel" or are not based on the promising or evidence-based practices that have emerged through research, evaluation, and hard-won experience. To ensure that projects don't expend unnecessary effort where lessons have already been learned, there is great opportunity-and even need-for dispersed projects and practitioners to learn and collaborate across boundaries of all kinds. Associations can serve a critical role in convening practitioners for large-scale networking events such as conferences or small-scale sessions such as charrettes or workshops where key ideas can be advanced. Associations publish peer-reviewed journals, trade magazines, newsletters, and other forms of membership communication, often with the dual purpose of sharing information from the field to create community and to translate research findings for practitioners. Associations also can provide listservs and online platforms where practitioners share innovations, find collaborators, ask questions, and discuss cross-cutting ideas. In membership organizations that are run mostly by dedicated volunteers, the vitality of such activities can depend upon the vitality of the community, as individuals and institutions have a choice about their degree of involvement

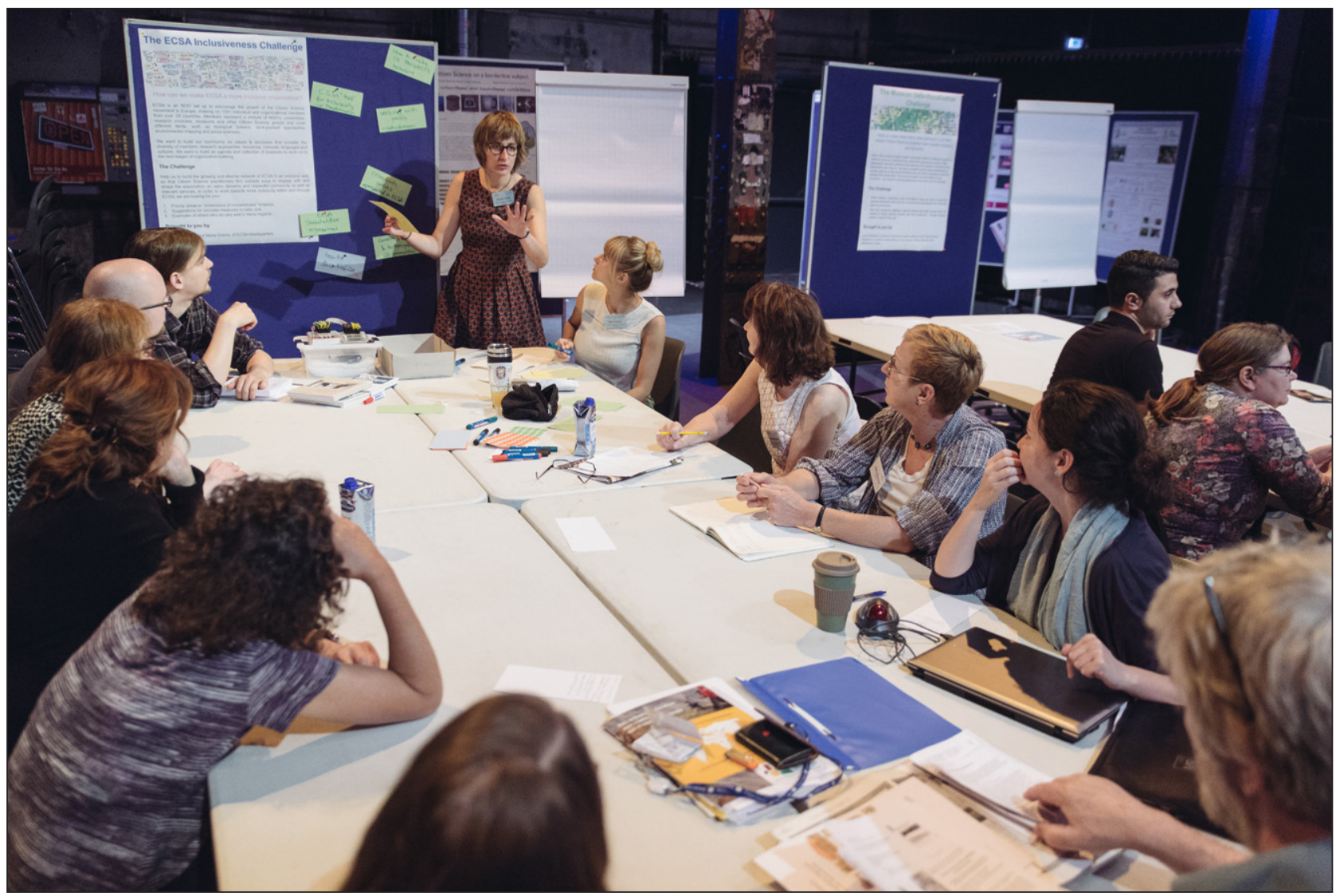

Participants engaged in breakout groups at the first international conference of the European Citizen Science Association-Citizen Science-Innovation in Open Science, Society and Policy-held in Berlin, Germany, May 2016. UFZ/ Florian Pappert. 
and engagement. But with intentional facilitation, all of the above activities can contribute to forming a community of practice by fostering connections across institutions and disciplines, and all can inform effective use of citizen science initiatives, maximize the impact of innovations, and avoid duplication of efforts.

In confronting common challenges and building on experiences, the citizen science community is establishing new norms and procedures that evaluate, validate, and reflect upon its own enterprise. For example, international and cross-disciplinary working groups are currently exploring and advancing a common language and definitions around citizen science, which help bring clarity to how and why citizen science is employed in any given project. Working groups are also engaging with challenges such as ethical considerations of collaborative work between paid and non-paid contributors, issues related to privacy, the intersection of citizen science and policy, and issues of inclusion, equity, and fairness within the larger citizen science field. Such targeted groups collaboratively interpret evidence stemming from research and evaluation as well as from "the wisdom of practice" to draft guidelines, recommendations, or status reports that can inform the work of individual practitioners or the field as a whole. These self-organized activities indicate and foster reflective professional practice and are essential for establishing citizen science as a field of practice that can be recognized and appreciated as a concept with meaning and value even outside of its own community. By formalizing such concepts and their associated norms and practices through targeted workshops, position statements, and recognition of promising practices or outstanding contributions, associations can advance and uphold exemplary work to lead and inspire others.

For scholarship around citizen science: Associations can play a significant role in advancing and sharing scholarship and the knowledge of practice to support the growth of the field, both for practitioners and for peers. Citizen science has proven a compelling venue for studying a practice in which some traditional boundaries around scientific research are re-thought and questions are raised that have both practical and scholarly implications for the field. Philosophical and structural challenges to research are common across projects that involve volunteers at any level. Citizen science shakes up the traditional science paradigm of how we produce knowledge, who is legitimate in doing science, what counts as research, who "owns" data or can legitimately use them, and who should be credited with findings. Citizen science also invites explicit reflection on how science intersects with societal concerns, because its practice can be seen as a means to embed science more deeply and more imminently into democratic and pluralistic societies. Citizen science is even forcing re-evaluation of how the larger scientific endeavor operates. For example, citizen science is linked to calls for open data (and free and open-source analysis tools) as well as to open access publication to enable those who contribute to research to access the fruits of joint efforts. Many if not most projects stay within a format in which scientists lead research projects with a "crowd" of contributors. Citizen science as a concept, however, also challenges well-established norms of the last two centuries relating to who has access to participate in scientific investigations and produce scientific knowledge. Even though a scientist has been defined traditionally by academic degree and source of income more than by skill, ability, interest, engagement, or activity, this may change as the impact of citizen science grows.

In addition to providing a venue for scholarship, such as with this journal, citizen science associations can take a leadership role in confronting and responding to broadreaching and cross-disciplinary concerns. These can include navigating the relationship between professional science and citizen science, addressing questions around equity, inclusion, and participation, or advancing our conceptual understanding of the meanings, degrees, and ethics of "participation." For instance, attention to the practice of citizen science has brought acclaim but has added scrutiny. Those new to the practice of citizen science may be skeptical of its value because they have encountered poorly implemented projects or because they are not aware of evidence regarding the maturity and validity of the field, particularly proven practices for creating scientifically sound data. Even highly effective and scientifically valid citizen science is still being questioned, as is evidenced by a 2015 editorial in the journal Nature (18 August 2015). Associations can craft standards and messaging to help advocate for the merits of citizen science in disciplines where it is practiced-as a case in point, leaders of the three associations collaborated to write a thoughtful response to the Nature editorial, highlighting the sophisticated work that is undertaken by mature projects to ensure data integrity (http://citizenscience.org/2015/09/15/ citizen-science-community-responds-to-nature-editorial/).

For the culture of scientific research: Associations have a role to play in cultivating a scientific culture of reflectiveness by initiating and channeling debates on critical issues within the peer community and with external stakeholders. To do so, associations can reach out to and draw on insights from more experienced cross-disciplinary societies that have addressed similar issues, such as those in public participation and democracy or science, technology, and society (STS) studies. Citizen science associations can also partner and collaborate with other disciplinary professional societies to foster citizen science as "cross-cutting" practice, conceivably working closely with, for instance, citizen science working groups within other science societies, or by establishing such working groups within the associations for other science societies. Associations that focus on citizen science can provide messaging or professional development around the practice of citizen science within the professional societies of different scientific disciplines, because practitioners, potential practitioners, and peer reviewers of citizen science may have limited access to materials or opportunities outside of their own disciplinary settings. In so doing, citizen science associations can be a leading voice for citizen science and can foster mutual exchange and learning, bringing ideas from citizen science to other fields and ideas from those fields back to the citizen science community. Such connections also can help to ensure that scholarship about the field 
is grounded in and informing practice across the diverse disciplinary contexts in which it can be found.

\section{Regional associations, global collaborations}

Citizen science, as a subset of the larger scientific enterprise, may be subject to the ideal that science should be independent of political and cultural boundaries. However, associations working together on a global image of citizen science recognize that many very local considerations influence how citizen science is valued and implemented. Considerations can include cultural differences in the status and power of academics or experts versus nonacademics/non-experts, or the legal and political ramifications of using citizen science approaches to hold governments or industry accountable. Even the instruments, protocols, and procedures that have been established in one part of the world might require adaptation to local conditions elsewhere. Additional considerations include practical issues of language (most notably, the word "citizen" carries different cultural meaning in different parts of the world, even as the term "citizen science" is widely understood as a concept that is not tied to nationality), funding structures, and the distance necessary and/or possible to travel for networking with colleagues. Furthermore, citizen science as a concept and practice involves more than scientific research alone. Other aspects, such as science education and science learning in formal and informal settings, social movements, or scientist engagement with various publics, can all differ across national or cultural boundaries, therefore providing additional reasons for both regional support and international exchange.

The three emerging associations-each described in detail below-are beginning to provide a joint global perspective on citizen science through collaboration, cooperation, partnership, exploring synergies, and fostering the mutual exchange of ideas. Individually, these associations provide support for geographically and politically coherent communities. Together, we (authors of this paper, representing each of these associations) acknowledge that some services can be enriched by shared efforts.

The value of a global community already can be seen in efforts of individual projects that operate at a global scale. Projects with multi-national and cross-continental collaborations can serve a variety of purposes, such as affecting cross-boundary science at large geographic scales or maximizing online participation from any geographic area. But global collaborations are not restricted to projects that operate at large geographic scales. Collaborations also are advancing the field through the recognition and application of innovative tools in new locations. The European Environment Agency's NoiseWatch app has, for example, been independently adapted for use in China. A world-wide virtual community has emerged for "Do-It-Yourself" sensor development through the Public Laboratory of Open Technology and Science, demonstrating that knowledge of practice can be shared across boundaries. Collaborations also can advance international "soft" diplomacy while affecting large-scale research and education, such as with the US-based but internationally active GLOBE program that aims at increasing science literacy through student participation in research. Associations can play a role in facilitating such connections and collaborations across geographic and/or disciplinary boundaries, and in highlighting the unique impacts of such collaborative efforts.

In this landscape of global project collaborations, associations are working in concerted ways to increase the capacity and credibility of the larger citizen science community and field worldwide. This mimics similar efforts by the scientific community at large where national organizations are highly linked in international networks and communities to address common issues. Establishing similar collaborations among associations in the field of citizen science can serve to amplify regional efforts without deemphasizing regional concerns.

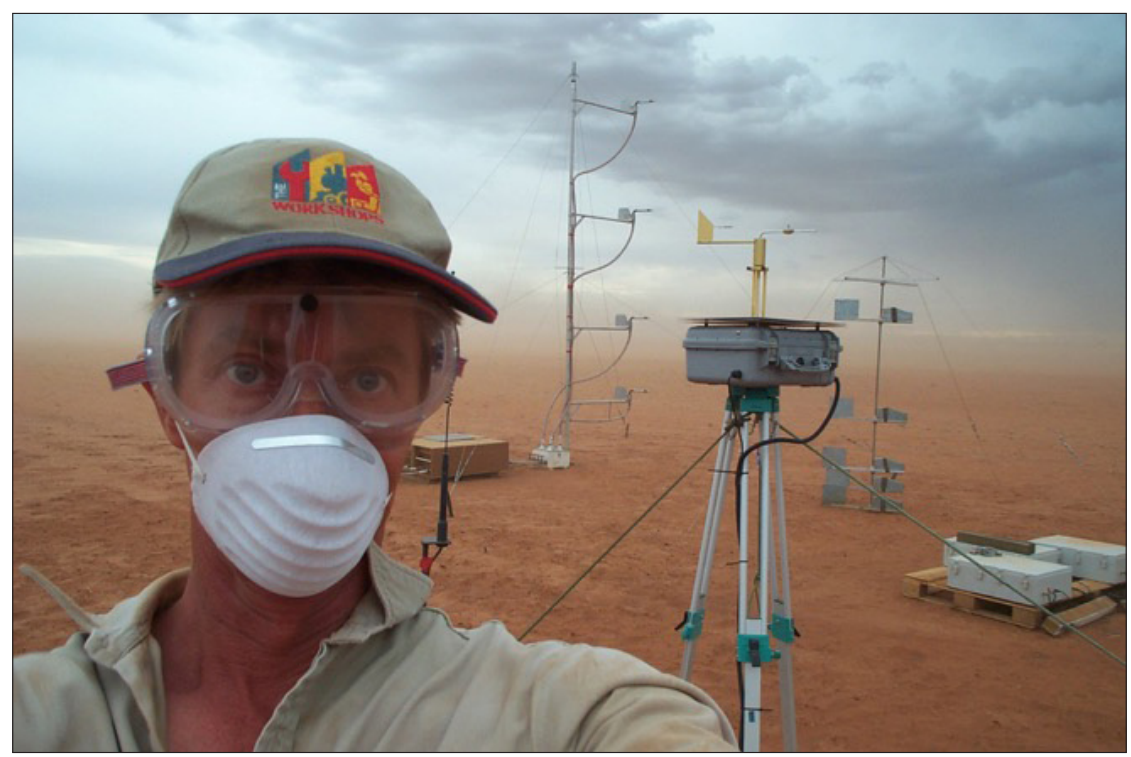

In 2015 ACSA members wrote an "Occasional Paper on Citizen Science" released by Australia's Chief Scientist at the country's inaugural citizen science conference in Canberra. The publication highlighted projects such as DustWatch, whose volunteers monitor storm activity using instruments such as DustTraks ${ }^{\circledR}$. (C) OEH/John Leys 
Working together has practical benefits for the emerging associations, for citizen science practitioners, and for the larger field. Collaborations among associations allow for greater efficiency and efficacy by dividing efforts and sharing resources in the development of knowledge, services, and infrastructure. Specifically, a joint global network can spearhead services such as sharing infrastructure-platforms, standards, data-and information on infrastructure and tools for citizen science (e.g., what software tools are available). With a clear, shared message, associations can liaise with disciplinary societies to understand how and to what degree citizen science is mainstreamed within those professional communities. Associations also can work together to link citizen science with related fields of study such as human-computer interaction or social psychology.

Strong ties among associations also can help to establish a unified global voice aimed at strengthening the awareness and reputation of citizen science within the larger scientific community and among members of the public. Relationships between associations can provide formalized avenues for learning and information sharing that foster mutual understanding of regional similarities (as well as differences), insights, and innovations, which can result from variation in existing citizen science projects, cultures, and politics. Together, associations can set agendas for global discussions and discourse around the value and contribution of citizen science for addressing global issues. Likewise, these groups can also facilitate or convene global or local citizen science projects that aim to address global problems. Taking advantage of this broad range of collaborative opportunities across associations can help to establish and expand the horizons of this emerging community and field. And institutionalized collaboration among regional associations will provide members of each who hold strong global identities as professionals or practitioners a sense of belonging to a global community, even if their institutional membership is with a regional association.

In September 2014, representatives of each association drafted a Memorandum of Understanding that establishes three focal areas for global collaboration. These are 1) scholarship via this journal, 2) regular joint conferences, and 3) online resources to facilitate networking and the sharing of best practices (http://ecsa.citizen-science. net/sites/default/files/mou-csa-ecsa-acsa_oct2016.pdf). Resources being scarce, working together allows us to share tasks, divide work, pool capacities, and learn from one another, all while benefiting from the diversity represented within the three associations and innovations based on cultural, linguistic, political, and geographic differences.

contd.

\section{Citizen Science Association}

\section{Meg Domroese, Abe Miller-Rushing, and Jennifer Shirk}

The Citizen Science Association (CSA), launched in February 2014, has more than 4000 members and increasingly serves as a communication hub for citizen science as well as for collective reflection on emerging challenges and for incubating innovations. More than 600 people attended the CSA's inaugural conference in February, 2015; more than 400 active subscribers share announcements and post questions to the discussion listserv; and this article is appearing in the second issue of the association's journal, Citizen Science: Theory and Practice. Coming together under the big tent of the Citizen Science Association has been an incremental process emerging from a diverse community of practitioners, and the continuing evolution of the field-and the association-will be driven by collaboration.

The idea of forming an association had been percolating for years. It was perhaps first voiced at a 2007 invitational meeting, funded by the US National Science Foundation and convened at the Cornell Lab of Ornithology (CLO), to develop a toolkit for citizen science project development. Questions arose there about the significance and the future of an emerging field, and Rick Bonney, CLO's Director of Public Engagement in Science, asked whether it was time to start thinking about an association (McEver et al. 2007).

Subsequent meetings continued to move the idea forward, such as the workshop on conservation outcomes of citizen science at the American Museum of Natural History in April 2011 (McEver et al. 2011). As different groups convened to discuss different aspects of the field-such as data management, policy concerns, and informal science learning--a growing sense that these discussions needed to be brought together became evident. In 2012, a nine-member organizing committee, with assistance from a large number of advisors and steering committee members, convened the first open conference on citizen science in the US, entitled Public Participation in Scientific Research (PPSR) 2012. Held in Portland, Oregon, the conference attracted 300 participants from a broad range of disciplines, levels of experience, and organization types, representing many countries and research on all seven continents. Besides the opportunity for participants at the conference to network and share experiences and challenges in citizen science, a goal of the conference was to affirm and initiate the formation of an organizational structure for citizen science as a field of study and practice (Benz et al. 2013). An external evaluator documented a growing sense among conference participants of community, identity, and professionalism in a highly interdisciplinary field, as well as the shared perception that citizen science was not sufficiently appreciated as a valid and valuable approach to science and public engagement (Heimlich 2012). 


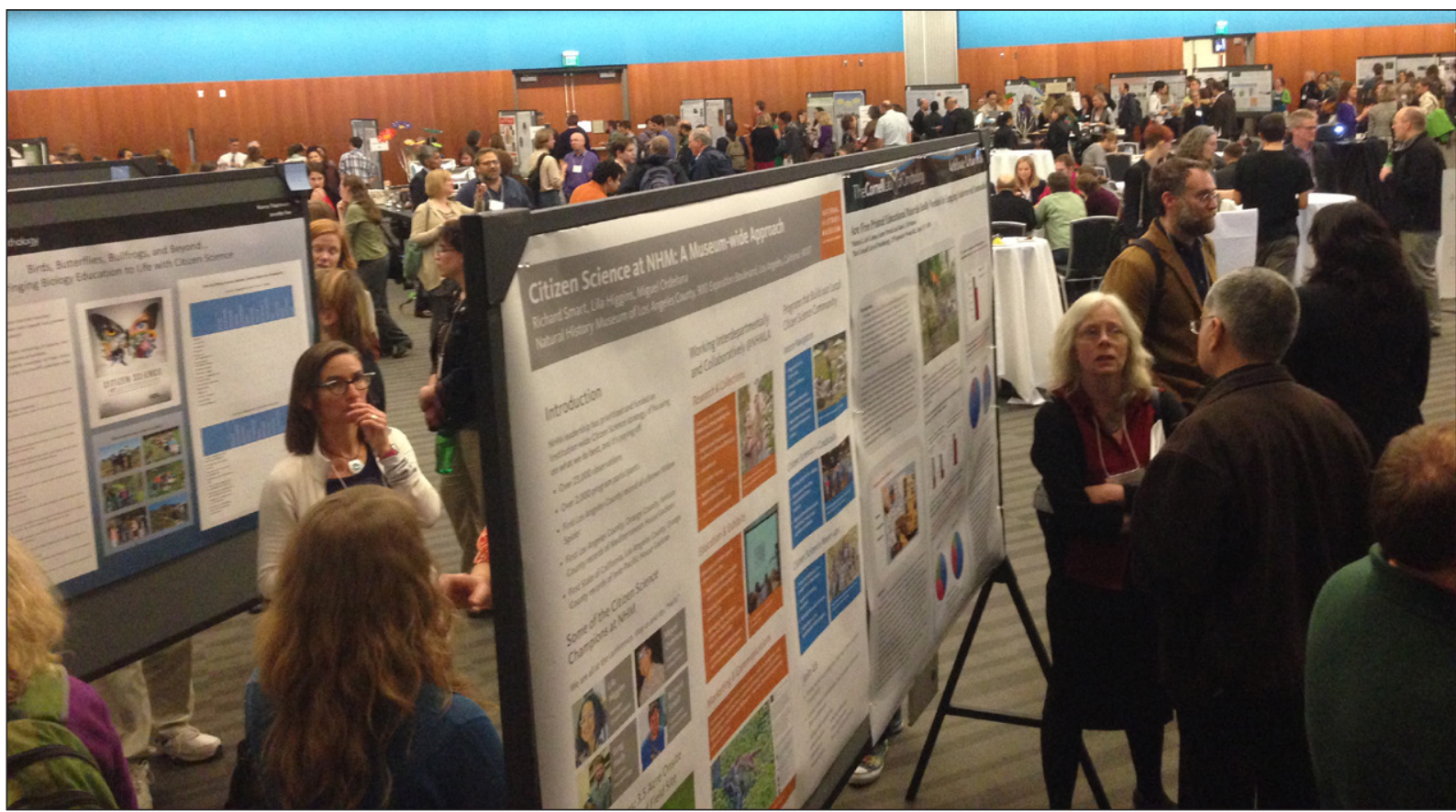

Hundreds of participants gathered at the poster session of the first Citizen Science Association meeting, held in San Jose, CA, February 2015. Natural History Museum of Los Angeles County/Citizen Science Office.

Conference participants enthusiastically affirmed the need for an association, and working groupsformed for discussion at the conference-continued afterward to develop the core functions necessary to establish the CSA. Committees for governance, web/ communications, journal, and conference each identified goals and established work plans. In a webinar in September 2013, representatives of these committees provided an overview of their work, solicited questions and feedback, and offered opportunities for others to get involved. By the end of 2014, committees included more than 80 members.

In January 2014, an interim steering committee was formed out of the governance committee to make decisions on behalf of the CSA prior to it becoming a legally recognized entity. This group committed to serving necessary governance functions, including work to ensure communication and coordination among the committees and to establish procedures for electing a board. This committee also outlined an initial membership structure that would engage the community's input and participation in the establishment of an association.

The CSA was launched in February 2014, with inaugural membership available free of charge. Interested individuals filled out a questionnaire to help the CSA better understand the diverse needs, interests, and expertise within the community. Respondents hailed from six continents and more than 65 countries. A great depth of experience was represented, with approximately $25 \%$ of respondents claiming sustained involvement in citizen science for more than four years (15\% for more than 10 years). At the same time there was evidence of a new and growing constituency, with another $25 \%$ more recently invested in citizen science activities. Most respondents claimed multiple roles in citizen science, including many as a volunteer contributor in addition to coordination and leadership roles. Many also noted involvement in multiple projects and research disciplines; projects represented disciplines as diverse as public health, bio-engineering, and history.

The CSA's inaugural members also voted on draft statements of the association's mission, vision, and goals, which the steering committee crafted based on input from PPSR 2012 conference participants and subsequent suggestions from the community. Important groundwork was thus laid for effective and open communication, vital to the CSA for supporting its members and the field.

Since the CSA's launch, the website CitizenScience.orgoriginally developed by the CLO-has been instrumental for presenting association goals and early activities. Moving forward, CSA is adopting a renewed version of CitizenScience.org which will increase capacity for web interactivity, including the opportunity to establish user profiles, share and evaluate resources, and participate in online discussions. CSA is taking a co-design approach to phasing in functionality for this platform to connect a global community of practitioners and to amplify the collection and delivery of information, resources, and avenues for constituents to become involved in the association. At the core will be a database of practitioners and their activities in the field, linked (through a collaboration with SciStarter) to a comprehensive database of projects. The CSA is 
also shepherding a collaboratively developed data exchange protocol and metadata standards ${ }^{1}$ (known as PPSR_CORE) that will efficiently allow sharing of project information across different online lists and communities.

Members elected a board of directors in December 2014 to lead incorporation and fundraising, as well as to provide oversight for the development of services for the field. Many of the functions and interests of the CSA, a primarily volunteer-based organization, are carried out through working groups. For example an education working group was established to identify opportunities and strategies to integrate citizen science in science, technology, engineering, and math (STEM) education, and this group led the education strand at the 2015 conference. Additional groups are advancing efforts in the areas of integrity, diversity, and equity (IDE), research and evaluation, professional development, data and metadata, and ethics. Most of these groups (including the editorial board for this journal) have an international membership, reflecting a commitment by the CSA to bridge conversations across associations and to develop services that support and engage a global community.

The CSA was incorporated in April, 2016, and the groundswell of interest is evidence of a lively community motivated to take the organization forward. Its second conference is planned for May 2017 in Minnesota, with more than 1000 attendees anticipated for workshops, talks, and public festival activities. And, inspired by CSA's involvement with US White House celebrations of citizen science, the first ever Citizen Science Day was celebrated in spring of 2016, an event which complements the bi-annual conference by encouraging local and regional sites to convene networking activities and to celebrate the significance and impacts of citizen science with practitioners and members of the public alike.

\section{European Citizen Science Association \\ Claudia Göbel, Katrin Vohland, and Muki Haklay}

In many European countries there is a long tradition of amateur scientists contributing to research, especially in the areas of birding, history, and astronomy. In fact, these traditions pre-date institutionalization of the sciences, and citizen engagement provided a leading role in structuring academia, including the co-evolution of those values which still can be found as good practice in science today.

Citizen science has seen a remarkable evolution, with Europe being a global hotspot for it in the $21^{\text {st }}$ century. The European Citizen Science Association (ECSA) helps catalyze connections across a growing community of actors engaged in participatory research across borders. The ECSA unites practitioners from research centers, universities, museums, non-governmental organizations, and the business sector to jointly advance the field. In seven working groups, ECSA members address key issues in citizen science: Communication and networking, quality standards and capacity building, data and IT infrastructures, and funding, as well as linking to decision makers. Key accomplishments resulting from the first two years of this work include a publication outlining ten principles of citizen science-a set of guidelines for good practice validated by the community (ECSA 2015a), and a growing collection of policy papers on pertinent issues in EU research and environmental policy (ECSA 2015b, c), as well as several workshops and conferences. ECSA is also advancing progress to improve citizen science data and metadata interoperability, in collaboration with the CSA effort mentioned above.
The impetus for forming a pan-European network for citizen science practitioners was provided by the Open Air Laboratories (OPAL) project in Great Britain. The OPAL project, a UK-wide citizen science initiative, links community science tightly to environmental education by conducting nature surveys. Starting in 2012, the idea to cultivate an exchange of knowledge and tools with other initiatives and stakeholders soon extended beyond the field of environmental monitoring and towards the whole of Europe. ECSA was launched officially during the European Union Green Week in 2013, and was formally registered under German law as a charity organization in April 2014. The formation of ECSA was strongly supported by science museums, which combine research, science communication, and education under one roof, and the ECSA headquarters is currently hosted at the Museum für Naturkunde Berlin (Museum for Natural History Berlin).

ECSA's major goal is to promote sustainable development via citizen science based on the idea that environmental sustainability is closely linked to research, innovation, and empowerment (ECSA 2015d). Citizen science is also seen as a potent contributor to policy implementation, for example, in the areas of environmental monitoring and open science. At the heart of ECSA's work is its role as a think tank for citizen science, supporting the exchange of ideas and experiences among practitioners as well as capacity building for project implementation and evaluation. Citizen science is understood as a flexible concept used for many forms of participatory research and engagement

contd. 
across the whole spectrum of scientific disciplines. The respect for this diversity of approaches is not only fundamental for the credibility of the association but a vital resource for a productive dialogue on improving citizen science methodologies. ECSA also establishes links to other stakeholder groups and strives to bundle the energies of its members to implement joint citizen science projects.

Since its foundation, ECSA has successively developed its community and organization. Four ECSA general assemblies - the main annual conferences of the association - have been held so far. The first meeting was held in April 2014 in Copenhagen in conjunction with the Living Knowledge Conference in order to set up basic structures of the organization, mainly the working groups. The second meeting was held in October 2014 in Berlin, following the EU BON stakeholder roundtable on citizen science and biodiversity data. At this second ECSA meeting, the ECSA board of directors was elected and the constitution was finalized. In October 2015, ECSA was invited to hold its third annual conference in Barcelona. Many projects and initiatives were presented at this meeting, and a vision for further development was discussed, which includes the relationship to concepts such as Responsible Research and Innovation (RRI) and the idea of decentralized thematic hubs as engines of the association. In addition, the ECSA advisory board was established. On the operative level, a steering committee has supported the executive board since January 2015 with a range of important decisions, including the development of a strategic plan (ECSA 2015d). The next big leap in terms of activity and impact was achieved through the first international ECSA conference in May 2016 in Berlin, with more than 300 attendees, where the international citizen science community addressed the ways in which participatory research generates innovations for science, society, and policy.

\section{Australian Citizen Science Association Jessica L. Cappadonna, Carla Sbrocchi, and Philip Roetman}

The Australian Citizen Science Association (ACSA) began with a group of individuals who recognized the need for a community of practice to support the expanding field of citizen science in Australia. A workshop was held in Brisbane in May 2014 to discuss the state of citizen science across Australia. The 90 attendees included project leaders, natural resource managers, educators, academic researchers, government representatives, and citizen scientists. An outcome of the workshop was strong support for a new, member-based, independent association, which would be equally valuable to organizations, managers of citizen science projects, and community volunteers.

The workshop spurred the formation of several working groups, tasked with progressing developmental aspects of such an association. A communications working group, for example, initiated channels of communication, including social media (Facebook, Twitter, LinkedIn, and Google+), an open-access electronic mailing list, and a website (www.citizenscience. org.au). A charter and objectives group developed a mission statement and a set of goals, values, and principles for the association. Members of an entity and governance working group developed the guidelines for a six-member establishment committee, which was elected in November 2014. This committee selected the Australian Museum as a host institution and finalised the association's name.

In July 2015, with the support of the Australian Academy of Science, Inspiring Australia, and numerous other sponsors, the association organized a national citizen science conference. Held in Canberra, the conference was attended by about 200 delegates representing a wide cross-section of individuals and organizations involved in citizen science across Australia, as well as delegates from CSA and ECSA. The event focused on the practice of citizen science and provided an exciting opportunity for the citizen science community to come together and discuss successes and challenges of the field. Australia's chief scientist, Professor Ian Chubb, provided a welcoming address and announced the release of a new paper focused on citizen science, indicating the growing high-level support for citizen science in Australia (Pecl et al. 2015). In conjunction with the conference, a meeting was held to formalise membership of the association and to elect a seven-member management committee. The conference and the election both very effectively allowed membership to take an active part in the association's development.

The ACSA Management Committee also finalised a three-year strategic plan in 2015, which operationalizes the association's vision, values, and goals, as well as provides indicators for success. The mission is to advance citizen science through knowledge sharing, collaboration, capacity building, and advocacy. The association aims to encourage broad and meaningful participation in citizen science through promoting inclusive and collaborative partnerships and facilitating a community that shares practices, knowledge, and tools to bring recognition to the value and impact of citizen science. 
Key focus areas for the next three years (2016-2018) include addressing capacity gaps within the field of citizen science and developing a long-term business model to ensure the association's sustainability. Committed federal funding has enabled ACSA to promote citizen science in Australia, focusing on the development of best practice, increasing engagement between the Australian public and scientific communities, and furthering partnerships.

\section{Conclusion: Toward an inclusive global community in support of citizen science}

Citizen science is, at once, a practice with a long history, a growing movement, a fledgling professional field, a global endeavor, and a powerful bridge between scientific research and the larger society that can benefit from it. In recognition of the increasing significance of citizen science, the three organizations described here are committed to working together closely, ensuring that an institutional bridge exists among all regions of the globe to share resources, tackle common issues, and facilitate co-learning. These three associations are not and will not be alone in this endeavor. Geographic networks of practitioners also are emerging in, for example, Austria, Brazil, Chile, China, Germany, India, New Zealand, and Spain. As opportunities arise, the three associations described here will work collaboratively with other regional and national organizations, helping to spawn new ones where none exist, and will form and nurture a global network that can represent the collective citizen science community in international settings such as the United Nations Environmental Programme (UNEP), the United Nations Educational, Scientific and Cultural Organization (UNESCO), or international climate negotiations (Conference of Parties or COP).

The collaborative nature of the three associations, however, is not limited to geographic scale. Communities of practice for citizen science are emerging in distinct disciplinary communities and within their professional associations, for instance within the Association of Science-Technology Centers, the British Ecological Society, or the Society for Conservation Biology. Furthermore, new and independent organizations are emerging that can benefit from formal connections to the larger citizen science community, such as the Great Barrier Reef Citizen Science Alliance and the US Federal Community of Practice for Crowdsourcing and Citizen Science. Connecting with and supporting these and other like-minded groups with robust conversations and exchange of ideas can enrich a global cross-disciplinary endeavor that cherishes all forms of scientific research, science learning, and engagement aligned with the concept of citizen science. We therefore welcome other organizations, large and small, to join in as we expand and enrich the notion of a global, cross-disciplinary community for citizen science.

\section{Acknowledgements}

The authors wish to thank the members of this community for being a driving force behind innovations in citizen science and the growth of this field. These associations would also not be possible without the individuals who have provided volunteer time and the organizations that have offered in-kind support to establish association infrastructure and services to advance the field. We would also like to acknowledge the many organizations that have invested in the development of these three associations, including (but not limited to): the National Science Foundation, National Geographic, Schoodic Institute, Cornell Lab of Ornithology, Inspiring Australia (an Australian Government Initiative), the Scottish Environmental Protection Agency, the Museum für Naturkunde Berlin, the Institute of Marine Science In Barcelona (ICM-CSIC), and other public and private organizations. The findings and conclusions in this report are those of the authors and do not necessarily represent the views of the funding agencies or the Department of Interior or the US Government.

\section{Competing Interests}

The authors have no competing interests to declare.

\section{Note}

${ }^{1}$ Inspired and supported by DataONE, PPSR_CORE has been a collaborative effort of CitSci.org, SciStarter, CitizenScience.org, and the Wilson Center's Commons Lab, and has been informed by the US Federal Community of Practice. The CSA's Data and Metadata Working Group, which is taking over oversight of these standards, has broad representation across the three citizen science associations described in this article to ensure global utility.

\section{References}

Benz, S., Miller-Rushing, A., Domroese, M., Ballard, H., Bonney, R., DeFalco, T., Newman, S., Shirk, J. and Young, A., 2013. Workshop 1: Conference on Public Participation in Scientific Research 2012: An International, Interdisciplinary Conference. Bulletin of the Ecological Society of America, 94(1): 112-117. DOI: http://dx.doi.org/10.1890/00129623-94.1.112

ECSA, 2015a. 10 Principles of Citizen Science. Retrieved from: http://ecsa.citizen-science.net/engage-us/10principles-citizen-science (September 23, 2016).

ECSA, 2015b. ECSA Policy Paper \#1. Endorsing the German Green Paper on Citizen Science. Retrieved from: http:// ecsa.citizen-science.net/sites/default/files/ecsa policy_paper_1.pdf (September 23, 2016).

ECSA, 2015c. ECSA Policy Paper \#2. Endorsing the White Paper on Citizen Science for Europe. Retrieved from: http://ecsa.citizen-science.net/sites/default/files/ ecsa_policy_paper_2.pdf (September 23, 2016).

ECSA, 2015d. ECSA Strategy. Retrieved from: http://ecsa. citizen-science.net/sites/default/files/ecsa_strategy. pdf (December 13, 2015).

Heimlich, J.E., 2012. Public participation in scientific research: 2012 conference evaluation. Ohio State University 
Extension, Columbus, Ohio, USA. Available at: http:// www.informalscience.org/public-participation-scientificresearch-2012-conference-evaluation-0.

McEver, C., Bonney, R., Dickinson, J., Kelling, S., Rosenberg, K., and Shirk, J., (eds.), 2007. Proceedings of the Citizen Science Toolkit Conference. Cornell Lab of Ornithology, Ithaca, NY, June 20-23, 2007. Available at: http:// www.birds.cornell.edu/citscitoolkit/conference/tool kitconference/proceeding-pdfs/Full Proceedings.pdf.

McEver, C., Domroese, M., Sterling E., Bonney, R., Braus, J., Arengo, F., Shirk, J., Petty, R. and Toomey, A., (eds.), 2011. Proceedings of the Workshop, Engaging and Learning for Conservation: Public Participation in Scientific Research. American Museum of Natural History, New York, New York, USA. Available at: http://www. birds.cornell.edu/citscitoolkit/conference/ppsr2011/ workshop-proceedings/workshop-proceedings.
Nature, 2015 (20 August 2015). Rise of the citizen scientist. Nature, 524: 265. DOI: http://dx.doi.org/ $10.1038 / 524265 a$

Pecl, G.T., Gillies, C., Sbrocchi, C. and Roetman, P., 2015. Building Australia through Citizen Science Occasional Paper Series: Australian Government Office of the Chief Scientist, Canberra. Available at: http://www. chiefscientist.gov.au/2015/07/occasional-paperbuilding-australia-through-citizen-science/.

Shirk, J.L., Ballard, H.L., Wilderman, C.C., Phillips, T., Wiggins, A., Jordan, R., McCallie, E., Minarchek, M., Lewenstein, B.V., Krasny, M. and Bonney, R., 2012. Public participation in scientific research: A framework for deliberate design. Ecology and Society, 17(2): 1-29. DOI: http:// dx.doi.org/10.5751/ES-04705-170229

Stebbins, R.A., 1992. Amateurs, Professionals and Serious Leisure. Montreal, QC: McGill-Queen's University Press.

How to cite this article: Storksdieck, M, Shirk, J L, Cappadonna, J L, Domroese, M, Göbel, C, Haklay, M, Miller-Rushing, A J, Roetman, P, Sbrocchi, C and Vohland, K 2016 Associations for Citizen Science: Regional Knowledge, Global Collaboration. Citizen Science: Theory and Practice, 1(2): 10, pp. 1-10, DOl: http://dx.doi.org/10.5334/cstp.55
Submitted: 10 January 2016
Accepted: 16 June 2016
Published: 11 November 2016

Copyright: (c) 2016 The Author(s). This is an open-access article distributed under the terms of the Creative Commons Attribution 4.0 International License (CC-BY 4.0), which permits unrestricted use, distribution, and reproduction in any medium, provided the original author and source are credited. See http://creativecommons.org/licenses/by/4.0/.

] $\mathbf{u}[\quad$ Citizen Science: Theory and Practice is a peer-reviewed open access journal published by Ubiquity Press. 